\title{
Change detection for visual satellite inspection using pose estimation and image synthesis
}

\author{
Ryan L. Buffington ${ }^{a}$ and John E. McInroy ${ }^{a}$ \\ ${ }^{a}$ Electrical and Computer Engineering Department, University of Wyoming, \\ 1000 E. University Ave., Laramie, WY USA 82071
}

\begin{abstract}
Satellites are subject to harsh lighting conditions which make visual inspection difficult. Automated systems which detect changes in the appearance of a satellite can generate false positives in the presence of intense shadows and specular reflections. This paper presents a new algorithm which can detect visual changes to a satellite in the presence of these lighting conditions. The position and orientation of the satellite with respect to the camera, or pose, is estimated using a new algorithm. Unlike many other pose estimation algorithms which attempt to reduce image reprojection error, this algorithm minimizes the sum of the weighted 3-dimensional error of the points in the image. Each inspection image is compared to many different views of the satellite, so that pose may be estimated regardless of which side of the satellite is facing the camera. The features in the image used to generate the pose estimate are chosen automatically using the scale-invariant feature transform. It is assumed that a good 3-dimensional model of the satellite was recorded prior to launch. Once the pose between the camera and the satellite have been estimated, the expected appearance of the satellite under the current lighting conditions is generated using a raytracing system and the 3-dimensional model. Finally, this estimate is compared with the image obtained from the camera. The ability of the algorithm to detect changes in the external appearance of satellites was evaluated using several test images exhibiting varying lighting and pose conditions. The test images included images containing shadows and bright specular reflections.
\end{abstract}

Keywords: satellite inspection, change detection, pose estimation, scale-invariant feature transform, raytracing

\section{INTRODUCTION}

In order to maintain space situational awareness (SSA), it is necessary to continuously observe objects of interest in orbit of the Earth. These objects, which are denoted resident space objects (RSOs), include active satellites, space debris, and natural objects. In low Earth orbit and middle Earth orbit, it is possible to use a combination of radar and ground-based telescopes to discover and inspect objects. However, it is very difficult to inspect objects in geosynchronous orbit using ground-based radar or ground-based telescopes due to the extreme distances involved.

Space based sensors, such as inspection satellites, could be placed much closer to the RSOs. This would make it possible to obtain better images of the RSOs with simpler optics. However, the bandwidth between the space based sensors and the ground is limited. This bandwidth could be conserved by processing the images on the spacecraft to determine which images contain new information. Images which do not show a change in the appearance of an RSO do not need to be transmitted to the ground. This process of detecting changes between two or more images is called change detection.

Performing change detection in the space environment presents special problems due to the harsh lighting conditions. Satellites frequently have long protrusions such as sensors, antennas, and solar panels which cast shadows. The lack of an atmosphere or dust to scatter light results in an extremely low level of ambient light. This causes shadows to be extremely dark when compared to similar images taken on Earth. Many satellites are constructed of highly polished materials such as glass and metal which exhibit bright specular reflections. These conditions can cause change detection algorithms to register false positives.

Send correspondence to mcinroy@uwyo.edu

Sensors and Systems for Space Applications IV, edited by Khanh D. Pham, Henry Zmuda,

Joseph Lee Cox, Greg J. Meyer, Proc. of SPIE Vol. 8044, 80440A · @ 2011 SPIE

CCC code: $0277-786 \mathrm{X} / 11 / \$ 18 \cdot$ doi: $10.1117 / 12.884151$

Proc. of SPIE Vol. 804480440 A-1 


\section{THEORETICAL BACKGROUND}

\subsection{The Pinhole Model}

Assume that the aperture of a thin lens can be approximated as a pinhole with a diameter that approaches zero. Under this assumption, all of the rays passing through the lens must pass through the optical center, $o$. This is the pinhole camera model. ${ }^{1}$ The pinhole model generates an image on the image plane which is upside down with respect to the world. Without loss of generality, it is possible to imagine that the imaging plane is in front of the optical center so that the image is not upside down. This creates the frontal pinhole imaging model shown in Figure 1.

Assume a point $p$ in the world in front of the camera has coordinates $\mathbf{X}=[X, Y, Z]^{T}$ relative to the coordinate system with its origin at the optical center of the lens. The image of point $p$ is defined as the intersection of the imaging plane and the ray going between the optical center $o$ and point $p$. From the application of similar triangles to Figure 1, it is apparent that $p$ and its image, $\mathbf{x}=[x, y]^{T}$, can be related by the perspective projection given in Equation 1. The coordinates of $\mathbf{x}$ are given in the image plane coordinate system, and $f$ is the focal length of the lens.

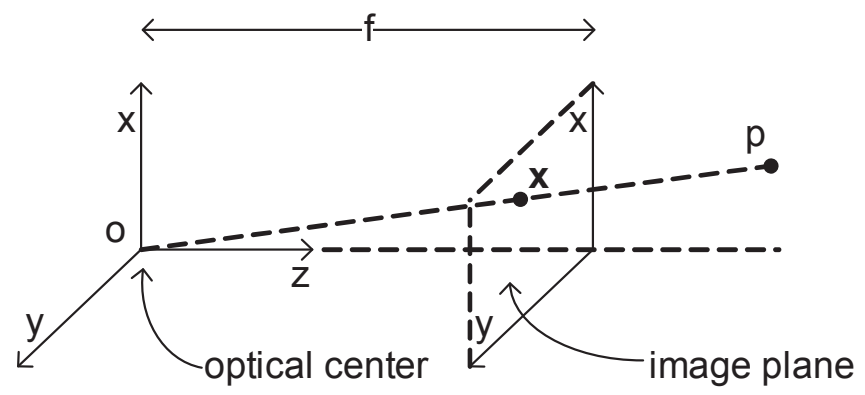

Figure 1. The frontal pinhole imaging model

$$
x=f \frac{X}{Z} \quad y=f \frac{Y}{Z}
$$

By definition, the set of all points which can can produce a given image of a point or a line is called the preimage of that point or line. For example, the preimage of point $p$ in Figure 1 is given by the points which lay along the ray passing through $x$ and $p$.

\subsection{The Perspective Transform}

It is possible to rewrite Equation 1 in homogeneous coordinates as given in Equation 2, where $\lambda$ is the depth of the point in the scene. The standard projection matrix $\Pi_{0}$ and ideal camera calibration matrix $K$ are defined in Equation 3. Other perspective transforms, such as those involving a nonplanar imaging surface, require a different form of the projection matrix. Also note that an uncalibrated camera will have additional terms in its $K$ matrix.

$$
\begin{gathered}
\lambda\left[\begin{array}{l}
x \\
y \\
1
\end{array}\right]=\left[\begin{array}{lll}
f & 0 & 0 \\
0 & f & 0 \\
0 & 0 & 1
\end{array}\right]\left[\begin{array}{llll}
1 & 0 & 0 & 0 \\
0 & 1 & 0 & 0 \\
0 & 0 & 1 & 0
\end{array}\right]\left[\begin{array}{c}
X \\
Y \\
Z \\
1
\end{array}\right] \\
K \equiv\left[\begin{array}{lll}
f & 0 & 0 \\
0 & f & 0 \\
0 & 0 & 1
\end{array}\right], \quad \Pi_{0} \equiv\left[\begin{array}{llll}
1 & 0 & 0 & 0 \\
0 & 1 & 0 & 0 \\
0 & 0 & 1 & 0
\end{array}\right] \\
\lambda \mathbf{x}=K \Pi_{0} \mathbf{X}
\end{gathered}
$$




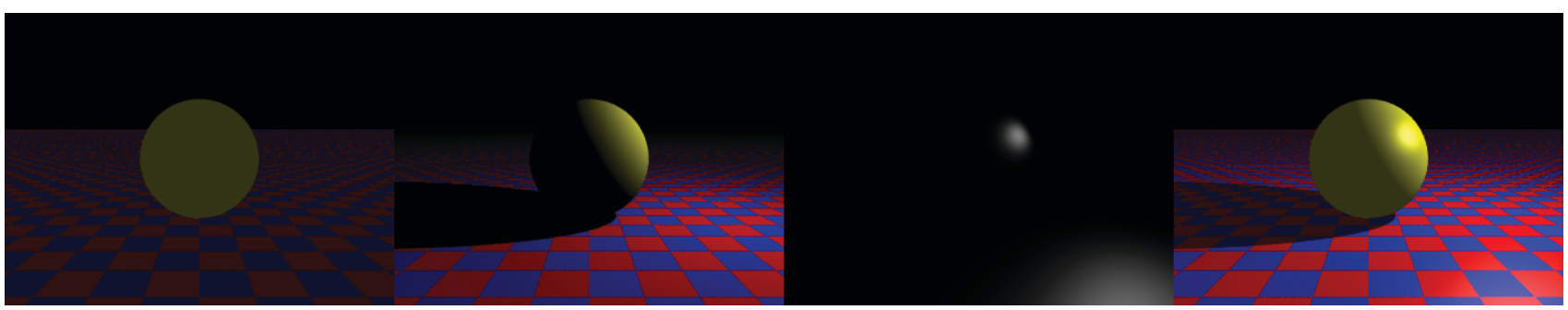

Figure 2. The Phong shading model

Normalizing the focal length of the camera so that $f=1$ produces the simplification given in Equation 5 . This simplification is the perspective transform for a calibrated camera. For simplicity, all further calculations will assume that camera calibration has occurred, and these calculations will use this model.

$$
\lambda \mathbf{x}=\Pi_{0} \mathbf{X}
$$

\subsection{Shading}

The intensity of a point in an image is a function of image lighting and the properties of the object at that point in the image. In the simplest object model, the intensity $I$ at a given image point $\mathbf{x}=[x, y]^{T}$ is the dot product of the vectors corresponding to the diffuse illumination $i_{d}$ and the reflectance coefficient $k_{d}$, as given in Equation 6. This is called the Lambertian reflection model. Information about the color and direction of the light are contained in the illumination vector, and the color and surface normal of the object are contained in the reflectance vector.

$$
I(x, y)=i_{d} \cdot k_{d}
$$

From this shading model, it is apparent that truly illumination invariant change detection from only $I$ is not possible. For example, a light gray object in dim lighting conditions could be replaced with an identically shaped dark gray object in bright lighting conditions, such that the same $I$ is produced.

To account for multiple light sources, ambient lighting, and specular reflections, a more complicated model, such as the Phong shading model ${ }^{2}$ is required. This model describes the appearance of an object as the sum of ambient, diffuse, and specular components. The ambient light term represents the low-intensity light which is scattered about the scene and has no apparent source or direction. Diffuse reflections are described using the Lambertian model. The specular component generates bright highlights on the object when a glossy surface reflects the light source like a mirror. The three components for an image of a sphere on a checkered plane, and the combination of these components into a final image is presented in Figure 2. From left to right, these images represent the ambient component, diffuse component, specular component, and the sum of the components.

\subsection{Dual view geometry}

The combination of rotation and translation which expresses the relationship between two coordinate systems is called the pose of the systems. Assume that two images of the same spacecraft have been taken from different poses, as shown in Figure 3. Point $p$ on the surface of the satellite may be expressed in the coordinate system attached to the first camera, $\left\{o_{1}\right\}$, the coordinate system attached to the second camera, $\left\{o_{2}\right\}$, or the coordinate system attached to the satellite, $\{o\}$. It is possible to express the relationships between the coordinate systems through the use of a rotation matrix and a translation vector. For example, converting from $\left\{o_{1}\right\}$ to $\left\{o_{2}\right\}$ is accomplished using Equation 7, where $X_{1}$ is point $p$ in coordinate system $\left\{o_{1}\right\}$, and $X_{2}$ is $p$ in system $\left\{o_{2}\right\}$. The matrix $R_{21}$ is the rotation matrix from $\left\{o_{1}\right\}$ to $\left\{o_{2}\right\}$, and vector $T_{21}$ is the translation vector from the origin of $\left\{o_{1}\right\}$ to the origin of $\left\{o_{2}\right\}$, expressed in $\left\{o_{2}\right\}$. Do not confuse $X_{1}$ and $X_{1}$ with $x_{1}$ and $x_{2}$, the images of point $p$ produced by projecting $p$ into the image planes using the perspective transform.

$$
X_{2}=R_{21} X_{1}+T_{21}
$$




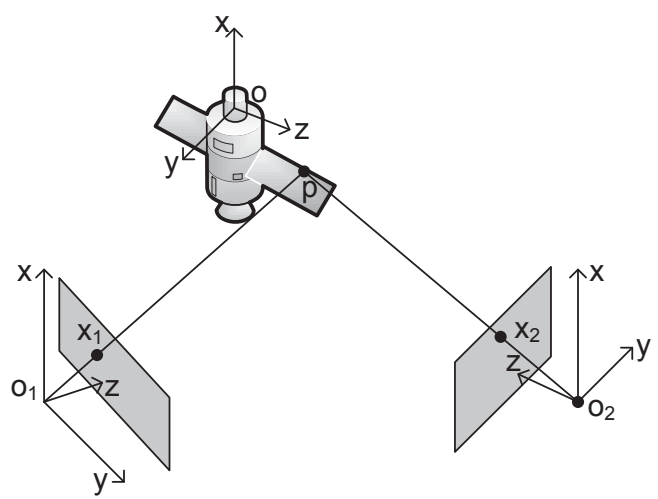

Figure 3. Dual view geometry

\section{THE OPTIMAL WEIGHTED 3D ERROR POSE ESTIMATION ALGORITHM}

\subsection{Derivation}

The optimal 3D pose estimation algorithm reconstructs a scene by minimizing the 3D position error for a given set of image correspondence points. The position error for a single set of correspondence points is shown as $e$ in Figure 4. In this figure, $u_{1}$ and $u_{2}$ are unit vectors pointing in the direction of the preimage of a correspondence point in image $I_{1}$ and $I_{2}$, respectively. The scalars $d_{1}$ and $d_{2}$ are the depths of the point in the scene. The location of correspondence point $i$ in camera $j$ can be expressed in 3D space using Equation 8.

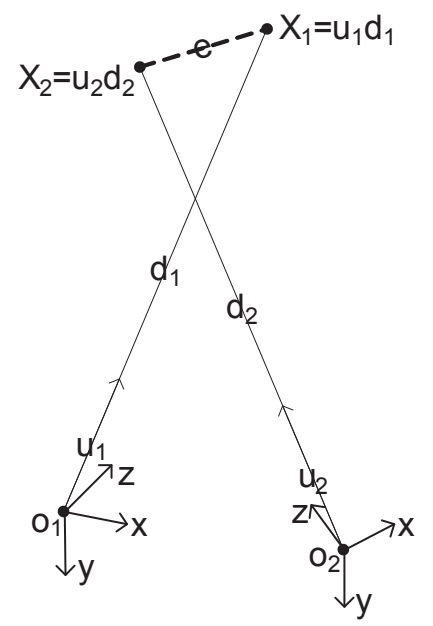

Figure 4. Graphical representation of position error, $e$

$$
X_{j}^{i}=u_{j}^{i} d_{j}^{i}
$$

Since points $X_{1}^{i}$ and $X_{2}^{i}$ are correspondence points, by definition they should describe the same point in 3D space. To equate these points, they must be transformed to be in the same coordinate system, as shown in Equation 9. The subscripts on $R_{21}$ and $T_{21}$ have been omitted for brevity.

$$
X_{2}^{i}=R X_{1}^{i}+T
$$

Substituting (8) into (9) produces Equations 10 and 11.

$$
u_{2}^{i} d_{2}^{i}=R u_{1}^{i} d_{1}^{i}+T
$$




$$
u_{1}^{i} d_{1}^{i}=R^{-1}\left(u_{2}^{i} d_{2}^{i}-T\right)
$$

Although $X_{1}^{i}$ and $X_{2}^{i}$ should describe the same point in 3D space, noise in the preimage vectors will create the 3D position error, $e^{i}$. Modifying (10) to include this error term produces (12). Ideally, $e^{i}$ will approach zero.

$$
\begin{gathered}
e^{i}=\left(R u_{1}^{i} d_{1}^{i}+T\right)-u_{2}^{i} d_{2}^{i} \\
e^{i}=\left[\begin{array}{ll}
R u_{1}^{i} & -u_{2}^{i}
\end{array}\right]\left[\begin{array}{c}
d_{1}^{i} \\
d_{2}^{i}
\end{array}\right]+T
\end{gathered}
$$

The superscript $i$ will now be dropped for simplicity. Matrix $R$ is a rotation matrix, therefore $R^{-1}=R^{T}$. To solve for the distances $d_{1}$ and $d_{2}$, let $k \equiv u_{1}^{T} R^{T} u_{2}$.

$$
\left[\begin{array}{l}
d_{1} \\
d_{2}
\end{array}\right]=\frac{-1}{1-k^{2}}\left[\begin{array}{cc}
1 & k \\
k & 1
\end{array}\right]\left[\begin{array}{c}
u_{1}^{T} R^{T} \\
-u_{2}^{T}
\end{array}\right] T
$$

Let $U \equiv\left[\begin{array}{ll}R u_{1} & -u_{2}\end{array}\right]$. Then substituting (14) into (13) produces a projection.

$$
\begin{gathered}
e=P T \\
P=I-\frac{1}{1-k^{2}} U\left[\begin{array}{cc}
1 & k \\
k & 1
\end{array}\right] U^{T}
\end{gathered}
$$

$P$ is a projection onto the subspace which is perpendicular to $u_{2}$ and $R u_{1}$. If $u \equiv \hat{u_{2}} R u_{1}$ then $P$ can be rewritten.

$$
P=\frac{u u^{T}}{\|u\|^{2}}
$$

The measurements of the preimages $u$ all contain angular errors $\theta$. Using the small angle approximation, the error in the $3 \mathrm{D}$ position of a point is $\theta d$, where $d$ is the distance to the point from the camera. Therefore, points which are farther away from the camera have more 3D error for a given angular error at the sensor.

As a result, points which are far away from the camera should be given less weight in the optimal estimate. Errors should be weighted with a term which is inversely proportional to $d$. This results in the definition of weighted error, $\left\|e_{w}\right\|^{2}$, given in Equation 18. The superscripts in this equation refer to exponentiation, not the index of the correspondence pair.

$$
\left\|e_{w}\right\|^{2}=\frac{\|e\|^{2}}{d_{1}^{2}+d_{2}^{2}}
$$

Taking the cross product of $u_{1}$ and $u_{2}$ with Equations 10 and 11 produces the following expressions for $d_{1}$ and $d_{2}$.

$$
d_{1}=\frac{-\hat{u}_{2} T}{\left\|\hat{u}_{2} R u_{1}\right\|}, \quad d_{2}=\frac{\hat{u}_{1} R^{T} T}{\left\|\hat{u}_{1} R^{T} u_{2}\right\|}
$$

Since $\left\|\hat{u}_{1} R^{T} u_{2}\right\|=\left\|\hat{u}_{2} R u_{1}\right\|=\|u\|$, the $d_{1}$ and $d_{2}$ terms can be combined.

$$
d_{1}^{2}+d_{2}^{2}=\frac{\left\|\hat{u}_{2} T\right\|^{2}+\left\|\hat{u}_{1} R^{T} T\right\|^{2}}{\|u\|^{2}}
$$


$P$ is a projection matrix, so $P^{2}=P$. Therefore $e^{T} e=T^{T} P T$. Applying this to (18) and (20) produces the results (21) and (22).

$$
\begin{gathered}
\left\|e_{w}\right\|^{2}=\frac{T^{T} P T}{d_{1}^{2}+d_{2}^{2}}=\frac{T^{T} \frac{u u^{T}}{\|u\|^{2}} T}{\frac{\left\|\hat{u}_{2} T\right\|^{2}+\left\|\hat{u}_{1} R^{T} T\right\|^{2}}{\|u\|^{2}}} \\
\left\|e_{w}\right\|^{2}=\frac{T^{T} u u^{T} T}{\left\|\hat{u}_{2} T\right\|^{2}+\left\|\hat{u}_{1} R^{T} T\right\|^{2}}
\end{gathered}
$$

Re-introducing the superscripts for each correspondence pair $i$ creates the final form for weighted error (23).

$$
\begin{gathered}
\left\|e_{w}^{i}\right\|^{2}=\frac{T^{T} u^{i}\left(u^{i}\right)^{T} T}{\left\|\hat{u}_{2}^{i} T\right\|^{2}+\left\|\hat{u}_{1}^{i} R^{T} T\right\|^{2}} \\
u^{i}=\hat{u}_{2}^{i} R u_{1}^{i} \\
J^{\prime}=\sum_{i=1}^{n}\left\|e_{w}^{i}\right\|^{2}
\end{gathered}
$$

To find an optimal estimate of $R$ and $T$, use the cost function (25). Minimize $J^{\prime}$ with respect to $R \in \mathrm{SO}(3)$ and $T \in S^{2}$, where $\mathrm{SO}(3)$ is the special orthogonal group which is the set of all rotation matrices, and $S^{2}$ is the set of all points on the surface of a unit sphere located at the origin. Only the direction of $T$ can be calculated; its magnitude is not recoverable without knowledge of the distances between the correspondence points in 3D space. Since $\mathrm{SO}(3)$ is a space with three dimensions, and $S^{2}$ is a space with two dimensions, the cost function can be minimized using a numerical search over a five dimensional space. This process is summarized in Algorithm 1.

\footnotetext{
Algorithm 1 Optimal estimation of pose by minimizing weighted 3D error

Given a set of $n$ image correspondence points $x_{1}^{i}, x_{2}^{i}$, for $i=1,2, \ldots, n$, and $n \geq 5$, this algorithm estimates the pose $R, T$ between the camera coordinate systems, such that the $3 \mathrm{D}$ coordinates are related by $X_{2}^{i}=R X_{1}^{i}+T$. The algorithm minimizes the sum of the 3D errors which have been weighted by a factor inversely proportional to the distance between $X^{i}$ and the camera.
}

1. Define the vectors $u_{j}^{i}$ which point in the direction of the preimage of correspondence point $i$ in image $j$.

2. Define the $3 \mathrm{D}$ weighted error $\left\|e_{w}^{i}\right\|^{2}=\frac{T^{T} u^{i}\left(u^{i}\right)^{T} T}{\left\|\hat{u}_{2}^{i} T\right\|^{2}+\left\|\hat{u}_{1}^{i} R^{T} T\right\|^{2}}$

3. Define the cost function $J^{\prime}=\sum_{i=1}^{n}\left\|e_{w}^{i}\right\|^{2}$

4. Minimize $J^{\prime}$ with respect to $R$ and $T$, subject to $R \in \mathrm{SO}(3)$ and $T \in S^{2}$, using a numerical search.

\subsection{Reducing the Search Size}

Define the term $D_{i}$ as given in (26). Then the weighted error measurement from (23) can be simplified to have the form (27).

$$
\begin{gathered}
D_{i} \equiv\left\|\hat{u}_{2}^{i} T\right\|^{2}+\left\|\hat{u}_{1}^{i} R^{T} T\right\|^{2} \\
\left\|e_{w}^{i}\right\|^{2}=\frac{1}{D_{i}} T^{T} u^{i}\left(u^{i}\right)^{T} T
\end{gathered}
$$


Due to the limited field of view of cameras used for remote inspection, it is not possible for $D_{i}$ to significantly change. This can be shown by recognizing that $\left\|\hat{u}_{2}^{i} T\right\|=\left\|u_{2}^{i}|\|||T|\left|\sin \phi_{2}^{i}\right|=\left|\sin \phi_{2}^{i}\right|\right.$, where $\phi_{2}^{i}$ is the angle between $T$ and $u_{2}^{i}$. Similarly, $\left\|\hat{u}_{1}^{i} R^{T} T\right\|=\sin \phi_{1}^{i}$, when $\phi_{2}^{i}$ is the angle between $R^{T} T$ and $u_{1}^{i}$. Assuming that both cameras have a narrow field of view, both $\phi_{2}^{i}$ and $\phi_{1}^{i}$ are unable to change significantly. As a result, the term $D_{i}=\sin ^{2} \phi_{2}^{i}+\sin ^{2} \phi_{1}^{i}$ can be considered invariant.

Since the $D_{i}$ term can be assumed to be invariant, it is possible to neglect it from the cost function (25), simplifying the search needed to find $R$ and $T$. The cost function can be rewritten as (28), where $M_{s}(R)=$ $\sum_{i=1}^{n} u^{i}\left(u^{i}\right)^{T}$.

$$
J=\sum_{i=1}^{n} T^{T} u^{i}\left(u^{i}\right)^{T} T=T^{T} M_{s}(R) T
$$

Since $M_{s}(R)$ is a function only of $R$, it is possible to minimize $J$ with respect to $T$, subject to the constraint $\|T\|=1$. Therefore, the minimum value of $J$ is produced when $T$ is the eigenvector of $M_{s}(R)$ corresponding to its minimum eigenvalue. In order to minimize $J$, it is necessary to find $R$ which minimizes the minimum eigenvalue of $M_{s}(R)$. This may be found by conducting a numerical search over a sphere of diameter $2 \pi$. Once $R$ has been found, $T$ can quickly be determined by calculating the eigenvector corresponding to the minimum eigenvalue of $M_{s}(R)$. Conducting a search over the surface of a sphere and then calculating one eigenvector is significantly easier than conducting a full numerical search over a five dimensional space. This process is summarized in Algorithm 2.

\section{Algorithm 2 Approximate estimation of pose by minimizing a minimum eigenvalue}

Given a set of $n$ image correspondence points $x_{1}^{i}, x_{2}^{i}$, for $i=1,2, \ldots, n$, an $n \geq 5$, this algorithm estimates the pose $R, T$ between the camera coordinate systems, such that the $3 \mathrm{D}$ coordinates are related by $X_{2}^{i}=R X_{1}^{i}+T$. The algorithm minimizes the sum of the $3 \mathrm{D}$ errors which have been weighted by a factor inversely proportional to the distance between $X^{i}$ and the camera, assuming that the field of view of the camera is sufficiently narrow.

1. Define the vectors $u_{j}^{i}$ which point in the direction of the preimage of correspondence point $i$ in image $j$.

2. Define $M_{s}(R)=\sum_{i=1}^{n} u^{i}\left(u^{i}\right)^{T}$

3. Find the rotation matrix $R$ which minimizes the minimum eigenvalue of $M_{s}(R)$ by performing a numerical search over a sphere of diameter $2 \pi$.

4. $T$ is the minimum eigenvector of $M_{s}(R)$, subject to $\|T\|=1$.

\subsection{Performance}

The performance of the optimal algorithm, the minimum-eigenvalue algorithm, and the eight-point algorithm were compared by randomly generating ten noisy correspondence points which describe a randomly generated pose. The pose estimate was compared to the pose which was originally used to generate the correspondence points. The rotation error between $R$ and $R_{e s t}$ and the relative translation error between $T$ and $T_{e s t}$ were calculated for each case.

To calculate the rotation error, first define the vee operator, $\vee$. For a $3 \times 3$ skew symmetric matrix, the vee operator transforms the matrix into the $3 \times 1$ vector shown in Equation 29. Similarly, define the wedge operator $\wedge$ to transform a $3 \times 1$ vector into a $3 \times 3$ skew symmetric matrix, as shown in (30).

$$
\begin{gathered}
{\left[\begin{array}{ccc}
a_{11} & a_{12} & a_{13} \\
-a_{12} & a_{22} & a_{23} \\
-a_{13} & -a_{23} & a_{33}
\end{array}\right]^{\vee} \equiv\left[\begin{array}{c}
-a_{23} \\
a_{13} \\
-a_{12}
\end{array}\right]} \\
{\left[\begin{array}{l}
a_{1} \\
a_{2} \\
a_{3}
\end{array}\right]^{\wedge} \equiv\left[\begin{array}{ccc}
0 & -a_{3} & a_{2} \\
a_{3} & 0 & -a_{1} \\
-a_{2} & a_{1} & 0
\end{array}\right]}
\end{gathered}
$$


The rotational error in degrees between $R$ and $R_{e s t}$ is calculated using (31), where log refers to the matrix logarithm operator.

$$
R_{\text {error }}=\left\|\left(\log \left(R^{T} R_{e s t}\right)\right)^{\vee}\right\| \frac{360}{2 \pi}
$$

The error between $T$ and $T_{\text {est }}$ can be calculated by taking the magnitude of the difference between the vectors. Since the algorithms do not calculate the magnitude of the translation vector, $\left\|T_{\text {est }}\right\|=1$. Therefore it is necessary to make $T$ a unit vector as shown in Equation 32.

$$
T_{\text {error }}=\left\|\frac{T}{\|T\|}-T_{\text {est }}\right\|
$$

Random poses were generated by generating random $R$ and $T$ pairs. $R$ was generated by taking a random $3 \times 1$ vector, applying the wedge operator, and then applying the matrix exponential. A second random $3 \times 1$ vector was assigned to be $T$. The $3 \mathrm{D}$ locations for ten points in the scene were randomly selected. Points that did not satisfy the positive depth constraint were replaced until all ten points were located in front of both cameras. Images of the randomly located 3D points were generated using the perspective transform. Noise was added to these correspondence points, and the noisy correspondence points were passed to the pose estimation algorithms.

The process was repeated with varying amounts of noise added to the normalized image coordinates of the correspondence points. One thousand scenes were generated for each noise level. For each scene, all three algorithms were used to estimate the pose and the amount of $R$ and $T$ error was calculated and recorded. It is assumed that a rotation estimate with an angular error of less than five degrees is useful, and that a translation estimate with relative error of less than 0.1 is useful. The relative number of useful estimates for each algorithm is displayed in Figure 5.

As seen in Figures 6 and 7, the minimum eigenvalue method described in Algorithm 2 outperformed the other algorithms when the standard deviation of the noise was greater than 0.002 normalized image coordinate units. Although the optimal algorithm is optimal in the sense that it minimizes 3D errors, it does not necessarily minimize $R$ and $T$ errors. For this reason, it is possible for the eight-point algorithm to outperform the optimal $3 \mathrm{D}$ algorithm. Although it would be expected that the optimal 3D algorithm would outperform the minimumeigenvalue algorithm, since the minimum-eigenvalue algorithm is a simplified version of the optimal 3D algorithm, this was not the case. Both algorithms use a numerical search to find an optimal value, and do not have a closed form solution. Occasionally the numerical search would fail to converge to the actual minimum value of the respective cost function. Due to the increased complexity of the search required by the optimal 3D algorithm, this algorithm failed to converge more frequently than the minimum-eigenvalue algorithm, resulting in its inferior performance.

Unlike the eight-point algorithm which has $R$ and $T$ errors distributed near zero, the 3D optimal and minimum-eigenvalue algorithms have errors which are distributed farther from zero, as seen in Figures 6 and 7 . Since these errors are further from the actual value, they would be much easier to eliminate using random sampling and consensus (RANSAC) than the errors generated by the eight-point algorithm. They would be easy to detect, as they usually occur when the numerical search reaches its maximum number of iterations, resulting in a large value of the cost function $J^{\prime}$ or $J$.

\section{CHANGE DETECTION ALGORITHM}

The pose-based change detector takes two images of an RSO, $I_{1}$ and $I_{2}$, and estimates the pose between the two images, $R_{21}$ and $T_{21}$. The minimum-eigenvalue $3 \mathrm{D}$ algorithm, outlined in Algorithm 2, is used to perform this pose estimation. Assuming that the pose between the RSO and the base image is known, the pose between the base image and the comparison image can be transformed into the pose between the RSO and the comparison image. 


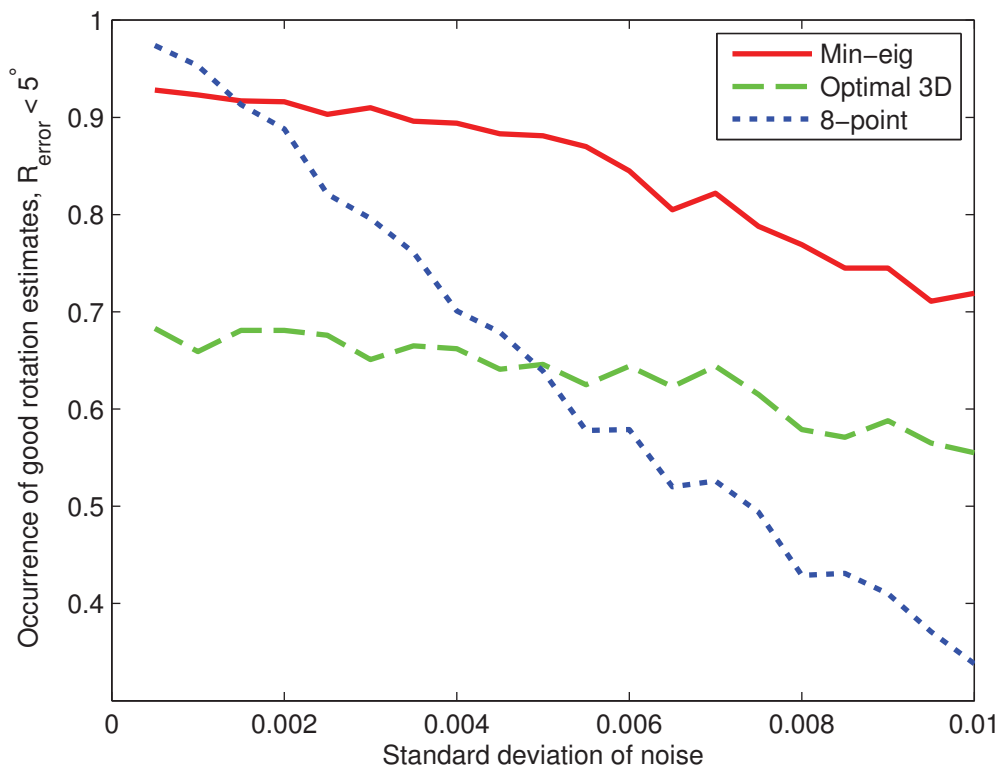

(a) Rotation error as a function of noise

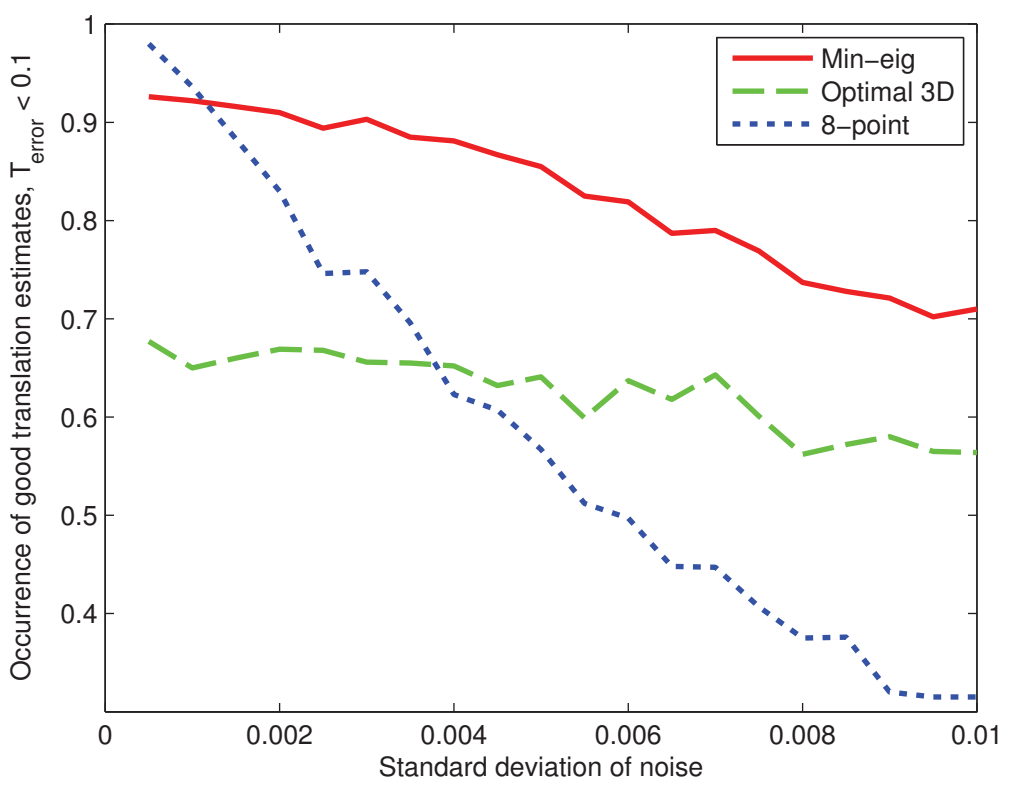

(b) Translation error as a function of noise

Figure 5. Performance of pose estimation algorithms in noise 


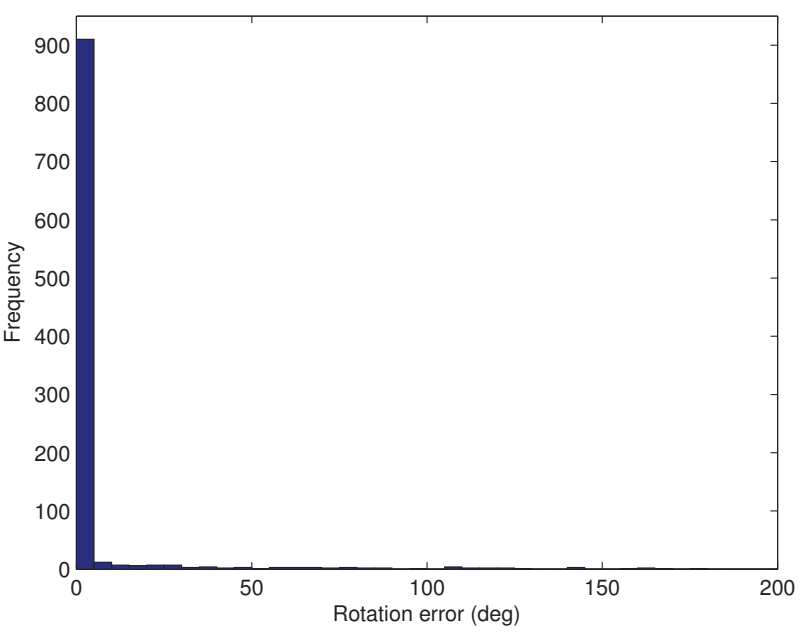

(a) Min-eigenvalue algorithm

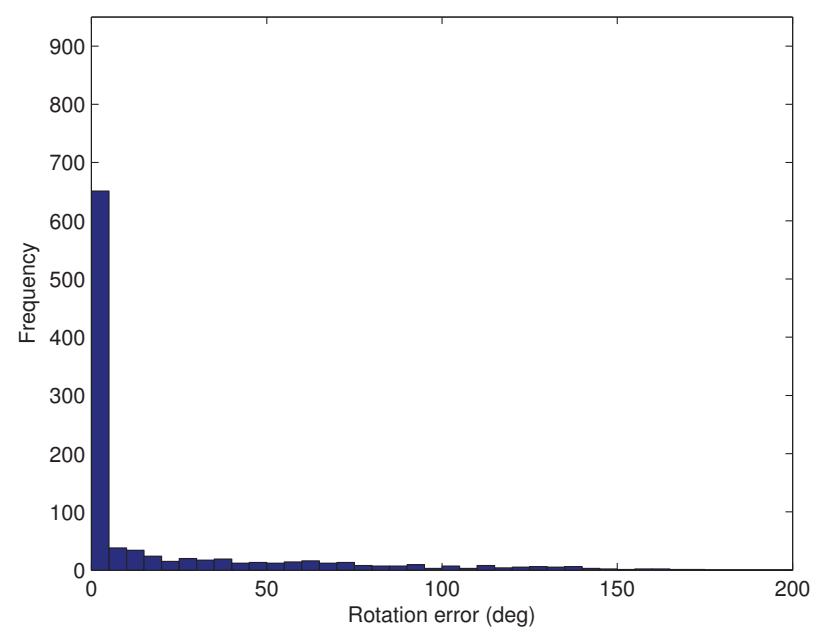

(b) Optimal 3D algorithm

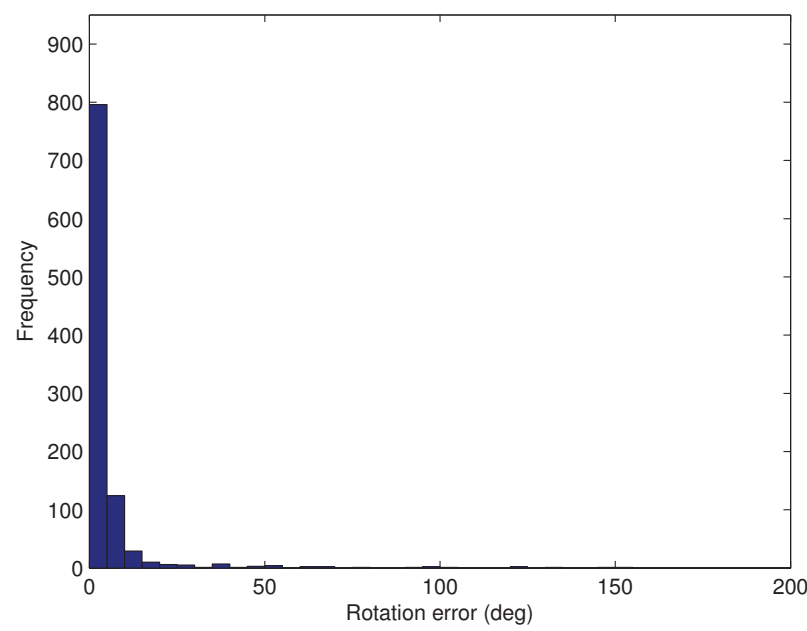

(c) Eight-point algorithm

Figure 6. Histogram of $R_{\text {error }}, \sigma=0.003$

Proc. of SPIE Vol. $804480440 \mathrm{~A}-10$ 


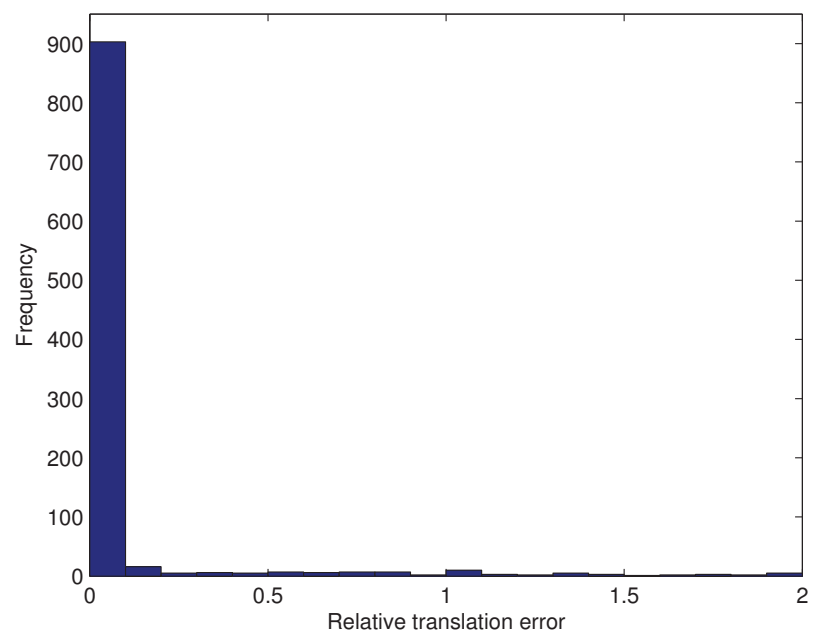

(a) Min-eigenvalue algorithm

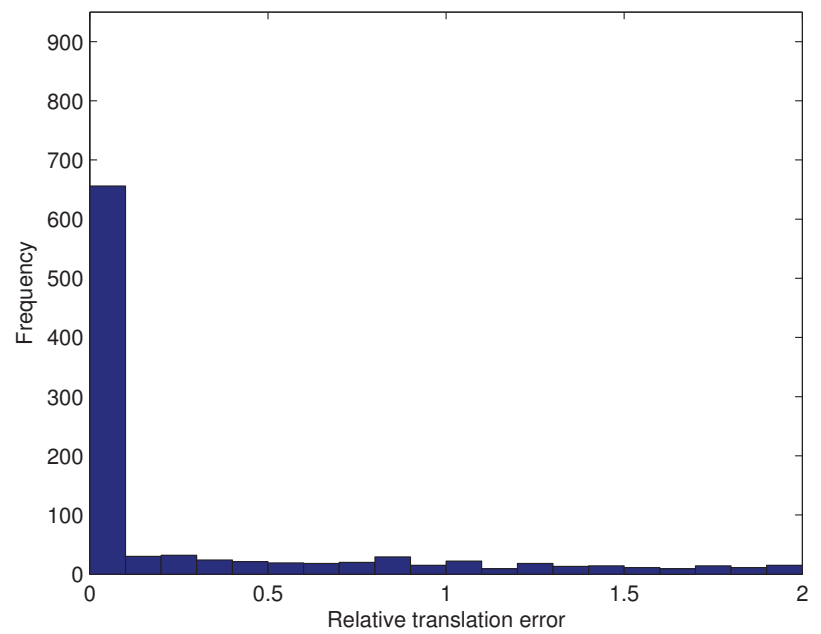

(b) Optimal 3D algorithm

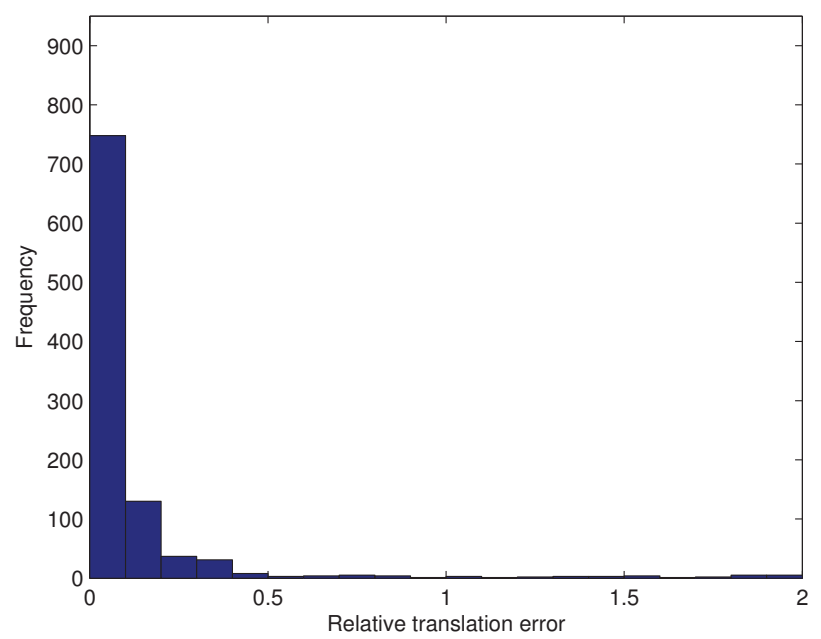

(c) Eight-point algorithm

Figure 7. Histogram of $T_{\text {error }}, \sigma=0.003$

Proc. of SPIE Vol. 8044 80440A-11 
It is assumed that a detailed 3D model of the RSO already exists, such as the CAD model used during its design, or a model generated from many images of the RSO. Since the pose between the inspection image and the RSO has been found, it is possible to use the model to generate $I_{3}$, a synthetic image which has the same pose as $I_{2}$. Finally, subtraction, thresholding, and morphological image processing are used to perform the comparison between $I_{2}$ and $I_{3}$.

\subsection{Pose Estimation}

The pose between the camera coordinate systems is described by a rotation from camera 1 to camera $2, R_{21}$, and a translation from camera 1 to camera 2 written in the camera 1 system, $T_{21}$. This pose is calculated using the process outlined in Algorithm 2. This algorithm requires a minimum of five correspondence point pairs to estimate $R_{21}$ and $T_{21}$. Correspondence points may be selected by hand or using SIFT.

Once the pose between camera 1 and camera 2 is known, it is necessary to calculate the pose between the RSO and camera 2. This is accomplished by using knowledge of the pose between the RSO and camera 1 . The estimated rotation from the RSO to camera $2, R_{20_{e s t}}$, can be found by first rotating into the frame of camera 1 , as shown in (33).

$$
R_{20_{e s t}}=R_{21_{\text {est }}} R_{10}
$$

Since the magnitude of $T_{12}$ is unknown, it is necessary to use other information about the scene to find the $T_{02}$ needed to generate the synthetic image, $I_{3}$. It is known that the camera looks at the center of the RSO, and it is also known that the camera always looks in the direction of its positive z-axis. Therefore, the direction of the vector from $\{o\}$ to $\{2\}$ can be found by rotating the vector $[0,0,-1]^{T}$ in the $\{2\}$ system so that it is expressed in the $\{0\}$ system. This can be accomplished using Equation 34 .

$$
T_{02_{\text {est }}} \propto R_{20_{\text {est }}}^{T}\left[\begin{array}{c}
0 \\
0 \\
-1
\end{array}\right]=R_{20_{\text {est }}}\left[\begin{array}{c}
0 \\
0 \\
-1
\end{array}\right]
$$

Assume that the distance between the RSO and camera 2 can be measured using another technique, such as a radar sensor or ephemeris data. Denote this distance by $\lambda$. The final estimate of $T_{02}$ is made by scaling $T_{02}$ est by $\lambda$.

$$
T_{02_{\text {est }}}=\lambda R_{20_{\text {est }}}\left[\begin{array}{c}
0 \\
0 \\
-1
\end{array}\right]
$$

\subsection{Image Generation}

The values of $R_{20_{e s t}}$ and $T_{02_{\text {est }}}$ are passed to the raytracer to generate $I_{3}$. Since the location of the Sun with respect to the the inspector is known from the appropriate ephemerides, it is possible to adjust the direction of the sun rays to render an image with appropriate lighting and shadows. It may be necessary to blur $I_{3}$ to match the diffraction and other imperfections in the imaging system used to obtain $I_{2}$.

\subsection{Morphological Filtering and Thresholding}

Images $I_{2}$ and $I_{3}$ are compared to perform change detection. A difference image is formed by subtracting the two images and taking the absolute value of the result. This image may contain long, thin errors caused by inaccuracies in the pose or lighting estimates. These errors are removed using the morphological erosion operator. A ball-shaped structuring element is used.

Finally a threshold is applied to the eroded difference image in order to identify areas which experienced a change. The gray level threshold is selected using Otsu's method. ${ }^{3}$ Changes are marked by white pixels in this image. 


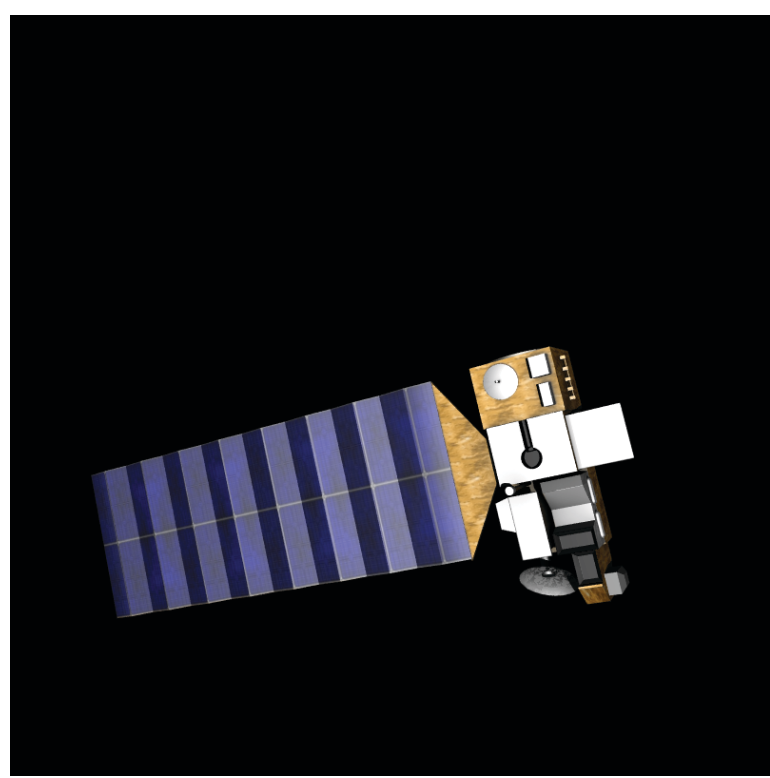

(a) Base inspection image $I_{1}$

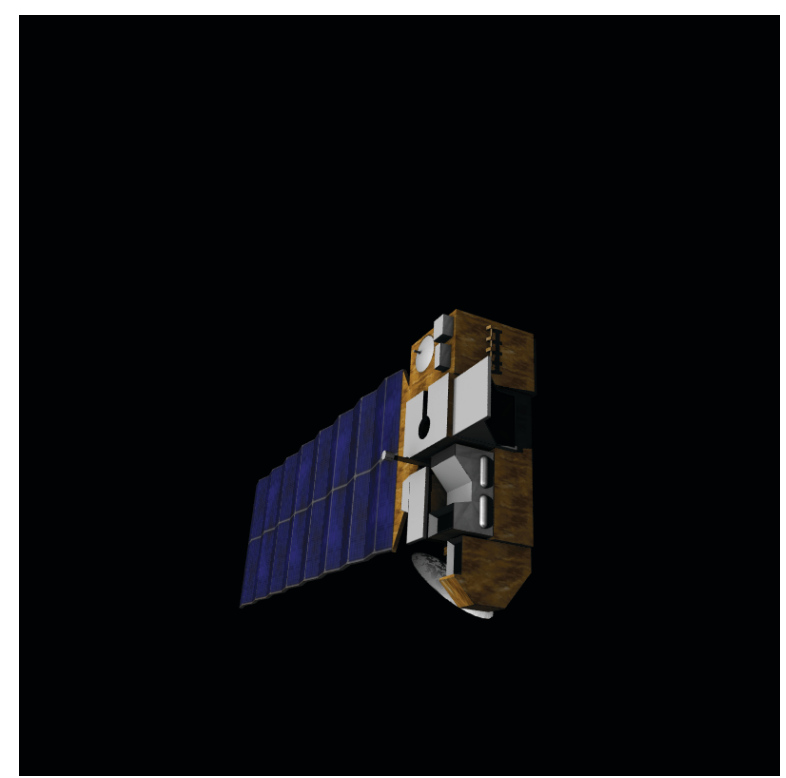

(b) Comparison inspection image $I_{2}$

Figure 8. Test images for pose algorithm

\section{SIMULATION}

To evaluate the performance of the algorithm, it was tested using the $3 \mathrm{D}$ model of the Aura satellite used to test the homography algorithm. Unlike the homography algorithm that only works for small rotation changes, this algorithm can be used for large changes in pose. Pose estimation algorithms are sensitive to correspondence point noise. To ensure that the results are repeatable, correspondence points were generated using knowledge of the actual pose and the perspective transform to project points on the RSO into the images. Under these ideal circumstances, the pose estimation algorithm is capable of making estimates of the rotation accurate to within 0.005 degrees and estimates of the translation direction accurate to one part in 10,000. For this simulation, additive Gaussian noise with a standard deviation of 0.75 pixels was added to the $\mathrm{x}$ and $\mathrm{y}$-components of the correspondence points.

The $800 \times 800$ images that were used to test the algorithm are presented in Figure 8, and the correspondence points and their reprojections are shown in Figure 9

The pose was successfully estimated with an error of 0.74 degrees. Since the translation vector, $T$, is only known up to a scaling factor, an appropriate translation vector is computed such that the center of the satellite is in the center of the field of view of the camera with the estimated rotation. The rendered image with the estimated pose is shown in Figure 10(a). The difference image obtained by subtracting $I_{2}$ and $I_{3}$ is presented in Figure 10(b). Note that the area which was occupied by the box is highlighted in this image.

The difference image was eroded using a ball-shaped structuring element with a radius of eight pixels. A larger structuring element was used because the RSO occupies more of the image frame than in the images used to test the homography-based method. Otsu's method was used to convert eroded difference image into the change detection result. The eroded difference image and change detection result are given in Figure 11. Note that the algorithm detected the absence of the box in $I_{2}$. Also note that the pose-estimator based algorithm was able to cope with a much larger change in pose than the homography-based algorithm, because it has access to information on the areas of the RSO which are occluded in the base image.

\section{RESULTS}

The pose-based change detection algorithm does an excellent job of detecting change in the presence of varying shadows and specular reflections, as shown in Figure 11. Since a comprehensive object and lighting model are 


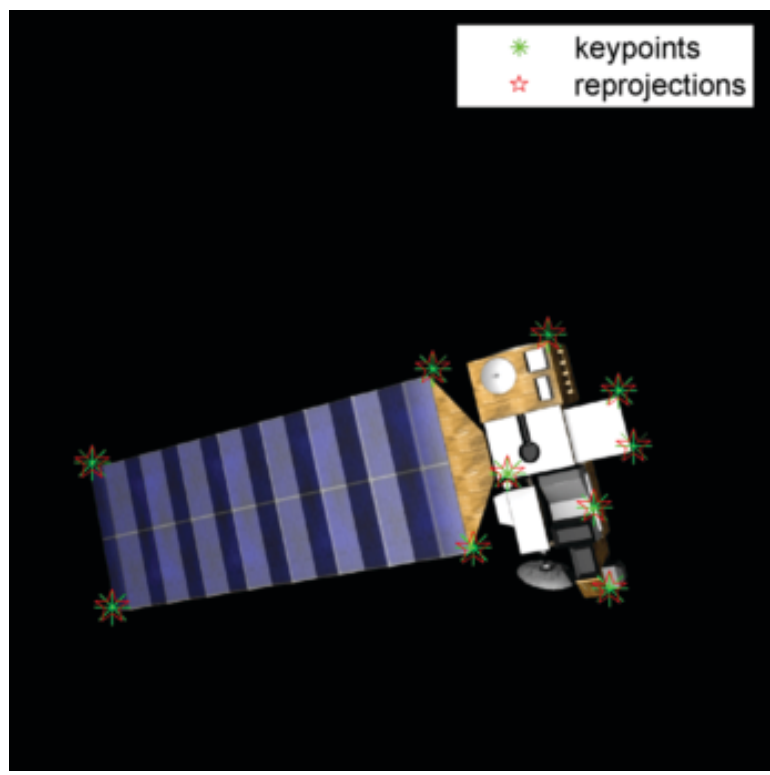

(a) Base image correspondence points

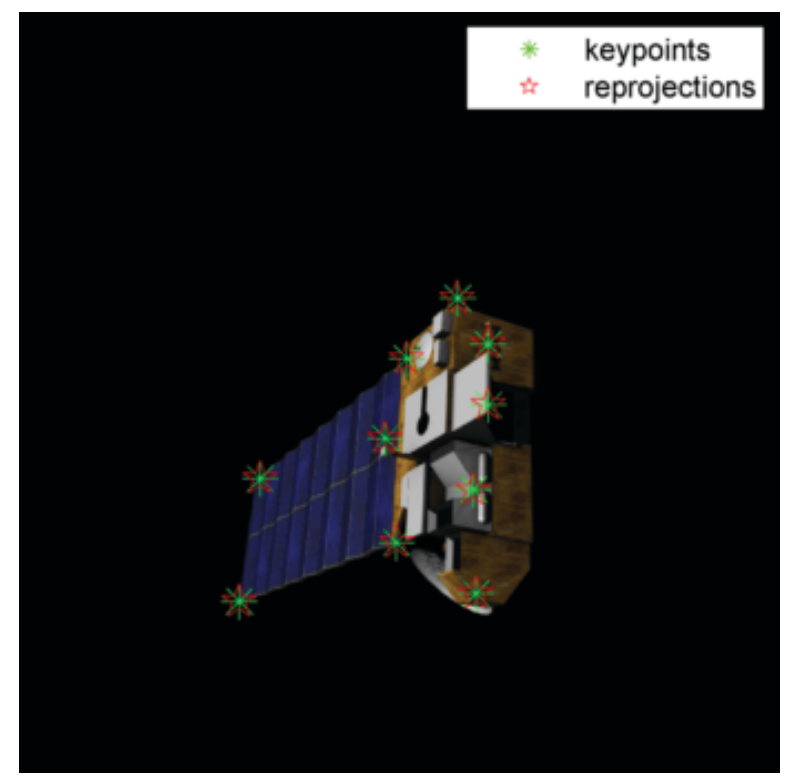

(b) Comparison image correspondence points

Figure 9. Pose algorithm correspondence points

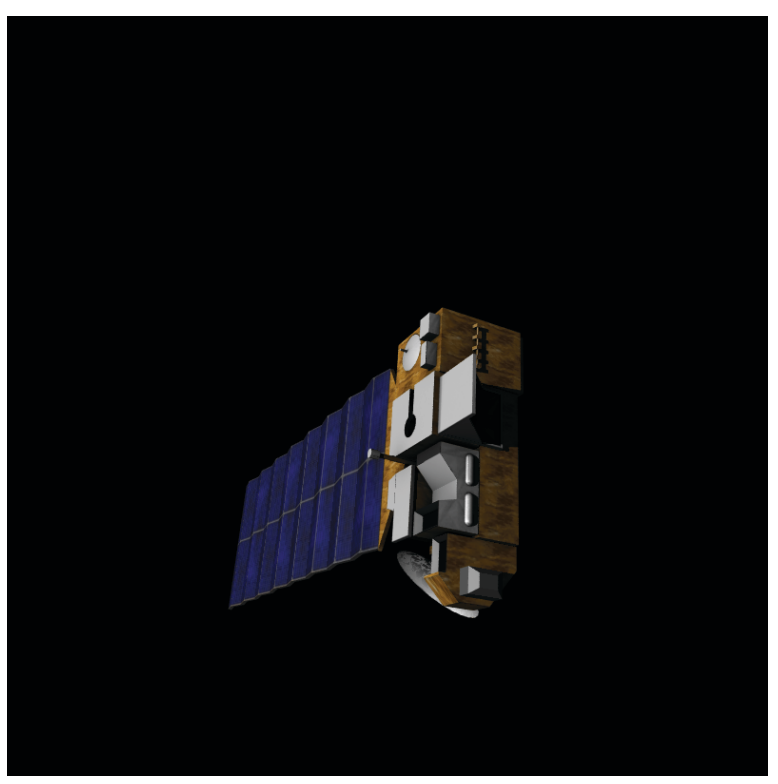

(a) Rendered image $I_{3}$

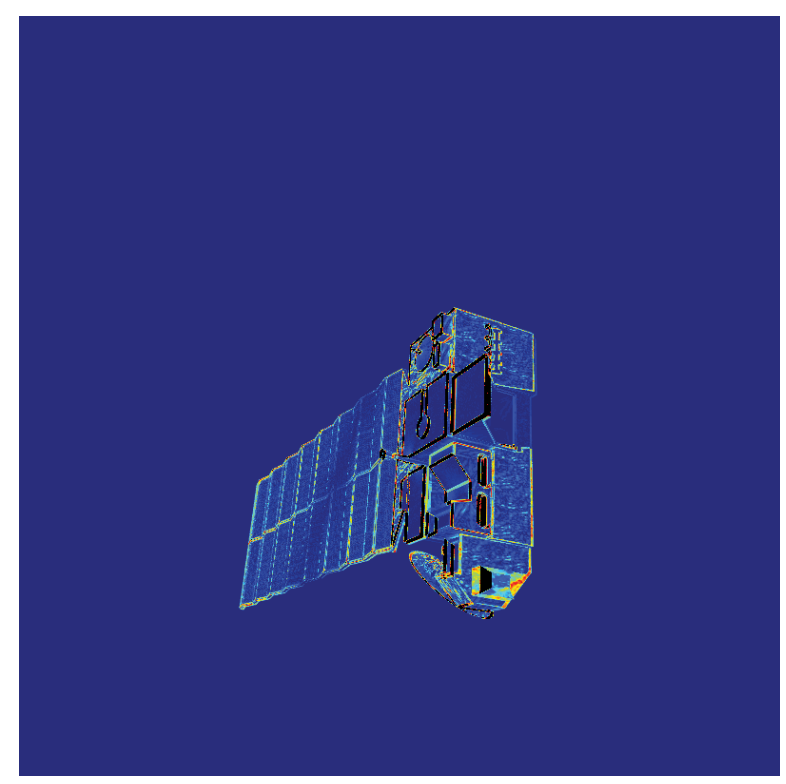

(b) Difference image between $I_{2}$ and $I_{3}$

Figure 10. Rendered and difference images 


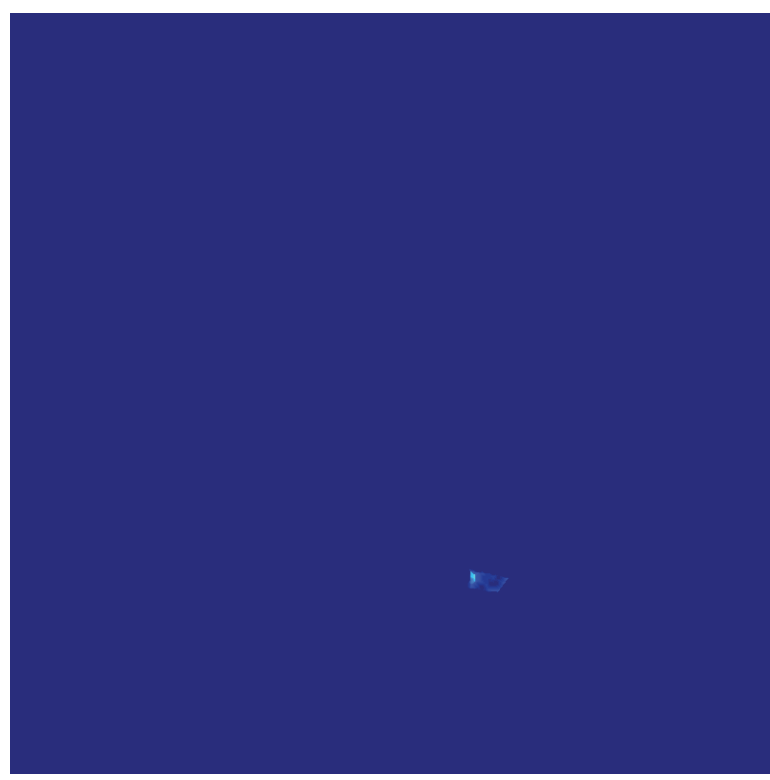

(a) Eroded difference image

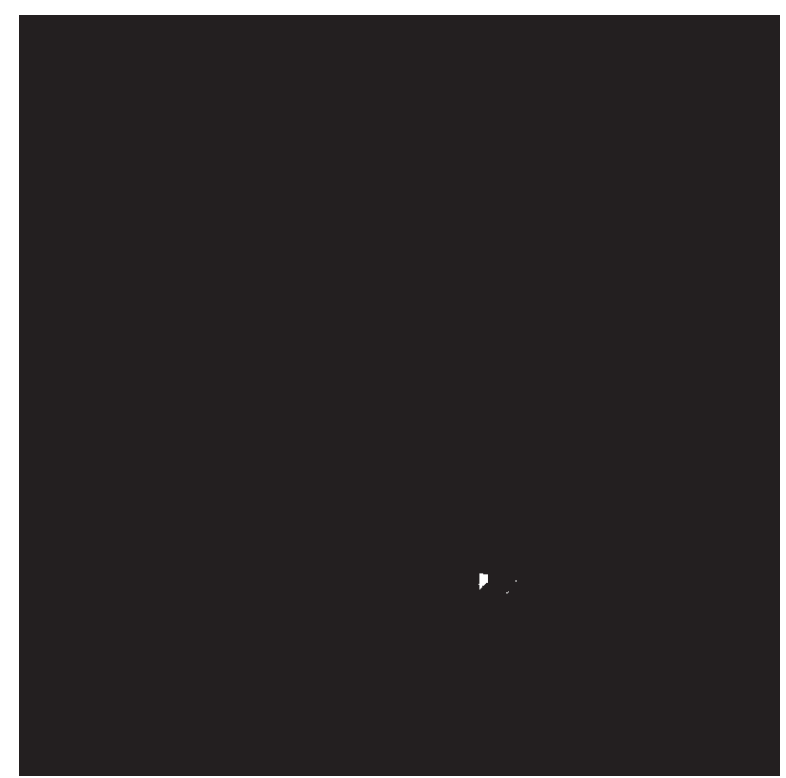

(b) Change detection result

Figure 11. Processing of difference image

used, the algorithm is both pose and illumination invariant. Unlike change detection algorithms which use a simple object model, the pose-based algorithm works in cases of extreme pose changes, as long as an accurate model of the satellite is known and accurate correspondence points are provided to the algorithm.

The minimum-eigenvalue algorithm was shown to produce usable pose estimates more frequently than the eight-point algorithm in the presence of correspondence point noise. The majority of its erroneous estimates are the result of problems with the numerical search. Due to the distribution of these errors, existing techniques such as RANSAC could be used to reduce the frequency of these estimation errors.

\section{REFERENCES}

[1] Ma, Y., Soatto, S., Košecká, J., and Sastry, S. S., [An Invitation to 3-D Vision], vol. 26, Springer (2006).

[2] Phong, B. T., "Illumination for computer generated pictures," Communications of the ACM 18, 311-317 (June 1975).

[3] Otsu, N., "A threshold selection method from gray-level histograms," IEEE Transactions on Systems, Man and Cybernetics 9, 62-66 (January 1979). 